\title{
Newborn genetic screening for hearing impairment: a population-based longitudinal study
}

\author{
Chen-Chi Wu, MD, PhD 1,2, Ching-Hui Tsai, MS3, Chia-Cheng Hung, PhD ${ }^{4,5}$, Yin-Hung Lin, MS ${ }^{1,4}$, \\ Yi-Hsin Lin, BS1, Fang-Li Huang, AuD1, Po-Nien Tsao, MD, PhD ${ }^{6}$, Yi-Ning Su, MD, PhD, ${ }^{5,7}$, \\ Yungling Leo Lee, MD, PhD ${ }^{3,9}$, Wu-Shiun Hsieh, MD ${ }^{6}$ and Chuan-Jen Hsu, MD ${ }^{1,10}$
}

\begin{abstract}
Purpose: The feasibility of genetic screening for deafness-causing mutations in newborns has been reported in several studies. The aim of this study was to investigate the long-term results in those who screened positive for deafness mutations; these results are crucial to determine the cost-effectiveness to justify population-wide genetic screening.
\end{abstract}

Methods: We performed simultaneous hearing screening and genetic screening targeting four common deafness mutations (p.V37I and c. $235 \mathrm{delC}$ of GJB2, c.919-2A>G of SLC26A4, and the mitochondrial $\mathrm{m} .1555 \mathrm{~A}>\mathrm{G}$ ) in 5173 newborns at a tertiary hospital between 2009 and 2015. Serial audiometric results up to 6 years old were then analyzed in children with conclusive genotypes.

Results: Newborn genetic screening identified 82 (1.6\%) babies with conclusive genotypes, comprising 62 (1.2\%) with GJB2 p.V37I/p.V37I,
16 (0.3\%) with GJB2 p.V37I/c.235delC, and 4 (0.1\%) with m.1555A>G. Of these, $46(56.1 \%)$ passed hearing screening at birth. Long-term follow-up demonstrated progressive hearing loss in children with the GJB2 p.V37I/p.V37I and p.V37I/c.235delC genotypes; this hearing loss deteriorated by approximately 1 decibel hearing level (dBHL) per year.

Conclusions: We delineated the longitudinal auditory features of the highly prevalent GJB2 p.V37I mutation on a general population basis and confirmed the utility of newborn genetic screening in identifying infants with late-onset or progressive hearing impairment undetectable by newborn hearing screening.

Genet Med advance online publication 16 June 2016

Key Words: deafness genes; GJB2; newborn genetic screening; sensorineural hearing impairment; universal newborn hearing screening

\section{INTRODUCTION}

Hearing impairment is the most common sensory defect in children. Approximately 1.9 per 1,000 children has bilateral permanent sensorineural hearing impairment $(\mathrm{SNHI})>40$ decibels hearing level (dBHL) at birth; this prevalence continues to increase during childhood and reaches 2.7 per 1,000 children before the age of 5 years and 3.5 per 1,000 during adolescence. ${ }^{1}$ Evidence shows that SNHI exerts a negative effect on speech, language, and cognitive development, ${ }^{2}$ and early identification and management are of paramount importance to improve the language, communication, mental health, and employment prospects of hearing-impaired children. ${ }^{3}$ The feasibility, cost-effectiveness, and benefits of universal newborn hearing screening (UNHS) for early identification of SNHI have been confirmed in several studies. ${ }^{4,5}$ However, current UNHS programs are limited by their ability to detect children with slight or mild SNHI whose hearing levels are less than the thresholds of the screening tools. ${ }^{6}$ Similarly, children with late-onset or progressive SNHI after birth cannot be identified, and these children may not benefit from improved outcomes conferred from early identification and intervention by UNHS.?
It is estimated that $\mathrm{SNHI}$ has a genetic cause in at least twothirds of children with the condition. ${ }^{8}$ Among a plethora of deafness genes identified to date, GJB2 (OMIM * 121011), SLC26A4 (OMIM ${ }^{\star} 605646$ ), and the mitochondrial $12 S$ rRNA gene (MTRNR1; OMIM $\left.{ }^{\star} 561000\right)$ are of special note from an epidemiological perspective; mutations in these three genes have been shown to be much more prevalent than other deafness genes across different populations. ${ }^{8}$ Patients with GJB2 (refs. 9,10) or SLC26A4 (ref. 11) mutations, or the common mitochondrial $12 \mathrm{~S}$ rRNA m.1555A $>\mathrm{G}$ mutation, ${ }^{12}$ might develop progressive SNHI with age. Moreover, it has been documented that some hearing-impaired patients with GJB2 (ref. 13) or SLC26A4 (ref. 14) mutations passed newborn hearing screening at birth.

Because genetic causes play a major role in $\mathrm{SNHI}$ in children, and because SNHI caused by certain genetic mutations may be undetectable by UNHS, we previously combined newborn genetic screening for common deafness mutations with newborn hearing screening in a pilot cohort. We demonstrated that newborn genetic screening might compensate for the inherent limitations of conventional UNHS by detecting subjects

\footnotetext{
${ }^{1}$ Department of Otolaryngology, National Taiwan University Hospital, Taipei, Taiwan; ${ }^{2}$ Department of Medical Genetics, National Taiwan University Hospital, Taipei, Taiwan; ${ }^{3}$ Institute of Epidemiology and Preventive Medicine, National Taiwan University College of Public Health, Taipei, Taiwan; ${ }^{4}$ Graduate Institute of Medical Genomics and Proteomics, National Taiwan University College of Medicine, Taipei, Taiwan; ${ }^{5}$ Sofiva Genomics Co., Ltd., Taipei, Taiwan; ${ }^{6}$ Department of Pediatrics, National Taiwan University Hospital, Taipei, Taiwan; ${ }^{7}$ Dianthus Maternal Fetal Medicine Clinic, Taipei, Taiwan; ${ }^{8}$ Department of Obstetrics and Gynecology, School of Medicine, Taipei Medical University, Taipei, Taiwan; ${ }^{9}$ Institute of Biomedical Sciences, Academia Sinica, Taipei, Taiwan; ${ }^{10}$ Department of Otolaryngology, Taichung Tzu Chi Hospital, Buddhist Tzu Chi Medical Foundation, Taichung, Taiwan. Correspondence: Yungling Leo Lee (leolee@ntu.edu.tw) Or Wu-Shiun Hsieh (hsiehws@ntu.edu.tw) or Chuan-Jen Hsu (cjhsu@ntu.edu.tw)
} 
with mild SNHI and those at risk for late-onset SNHI. ${ }^{15}$ Our findings concerning the feasibility and prognostic value of newborn genetic screening were subsequently validated in several studies. ${ }^{16-18}$ However, implementing population-wide genetic screening for deafness involves multiple issues and demands careful appraisal with regard to the potential psychosocial impacts on families and economic burden on the healthcare system. Longitudinal follow-up and analyses of clinical outcomes in newborns who screened positive for deafness mutations are necessary to address these issues.

\section{Subjects and newborn screenings}

\section{MATERIALS AND METHODS}

Between 2009 and 2015, 5,173 babies who received simultaneous newborn hearing screening and genetic screening for deafness were enrolled in this study. Newborn hearing screening was performed using distortion-product otoacoustic emissions (DPOAEs; GSI 60 DPOAE system, Grason-Stadler, Eden Prairie, Minnesota) before 2011; since 2012, the screening tool has shifted to automated auditory brainstem response (AABR; the ALGO3 screener, Natus, Pleasanton, CA) to comply with government policies. For newborn genetic screening, DNA samples extracted from heel-stick blood spots were subjected to mutation screening using a real-time polymerase chain reaction assay with fluorescence resonance energy transfer hybridization probes in a LightCycler 480 instrument (Roche, Basel, Switzerland). ${ }^{15}$ Genetic screening targeted the four most common deafness-associated mutations in the Taiwanese population: p.V37I (c.109G $>A$ ) and c.235delC of GJB2, c.919-2A>G of SLC26A4, and mitochondrial m.1555A $>\mathrm{G} .{ }^{19}$ In terms of the allele frequency in the hearing-impaired population, these four mutations amount to $>80 \%$ of the known deafness-associated mutations among Taiwanese individuals. ${ }^{19}$ Among these, GJB2 p.V37I, GJB2 c.235delC, and SLC26A4 c.919-2A>G are autosomal recessive mutations, whereas $\mathrm{m} .1555 \mathrm{~A}>\mathrm{G}$ is a maternally inherited mutation. Accordingly, subjects with two mutant GJB2 alleles (i.e., with the p.V37I/p.V37I, p.V37I/c.235delC, or c.235delC/c.235delC genotype); two mutant SLC26A4 alleles (i.e., with the c.919-2A $>\mathrm{G} / \mathrm{c} .919-2 \mathrm{~A}>\mathrm{G}$ genotype); or the m.1555A $>$ G mutation (either in homoplasmy or heteroplasmy) were interpreted as conclusive genotypes. Written informed consent for participation in the project was obtained from the parents of all infants, and all procedures were approved by the Research Ethics Committee of the National Taiwan University Hospital.

\section{Audiological assessments}

Infants with conclusive genotypes from genetic screening, despite their newborn hearing screening results, were referred to a pediatric otologist for comprehensive audiological assessments at 3 months. The comprehensive audiological assessments included behavioral testing (visual reinforcement audiometry) in a sound field using warble tones, narrow-band noise, and live voice; DPOAEs testing; and a diagnostic auditory brainstem response (ABR; Natus) under sedation to determine the hearing thresholds at $0.5,1,2$, and $4 \mathrm{kHz}^{20}$
After 3 months, we recommended a follow-up protocol-that the audiological assessments be conducted every 6-12 months-but this protocol can be adjusted depending on the children's auditory results and parents' request. For children with hearing thresholds $>40 \mathrm{dBHL}$, comprehensive audiological examinations including behavioral testing, DPOAEs, impedance testing, and ABR were scheduled for each follow-up evaluation, whereas for children with hearing thresholds $\leq 40 \mathrm{dBHL}$, only behavioral testing in a sound field was performed to avoid repeated sedations. For audiological follow-ups at 3 years and beyond, behavioral testing and ABR were replaced by pure-tone audiometry (GSI 61, Grason-Stadler, Eden Prairie, Minnesota) if the children were able to cooperate with the test procedure. The children's hearing levels were then determined by averaging the four-tone thresholds $(0.5,1,2$, and $4 \mathrm{kHz})$ of the better ear on $\mathrm{ABR}$ (children $<3$ years old) or pure-tone audiometry (children $\geq 3$ years old). For audiological follow-ups before 3 years for which $A B R$ was not performed, children's hearing levels were determined by averaging the four-tone thresholds on behavioral audiograms.

\section{Kaplan-Meier estimations}

Because all four common deafness mutations might be associated with progressive SNHI, hearing levels in children of different ages with conclusive genotypes were subjected to Kaplan-Meier analyses. Among the different genotypes, the numbers of audiological assessments in subjects with GJB2 p.V37I/p.V37I and GJB2 p.V37I/c.235delC fulfilled the requirement for performing Kaplan-Meier analyses. Two hearing levels of clinical significance-25 and $40 \mathrm{dBHL}-$ were set as the events. For the definition of end points, individuals with hearing levels of $25 \mathrm{dBHL}$ or more generally require adaptive listening strategies such as sitting closer to the source of sound, whereas individuals with hearing levels of $40 \mathrm{dBHL}$ or more generally need active treatment such as hearing aids (http://www.nidcd.nih.gov/ health/statistics/Pages/measuring.aspx). We used hazard ratios and two-sided 95\% confidence intervals to compare between genotypes using GJB2 p.V37I/p.V37I as the reference group. Testing was prespecified to occur between two mutant GJB2 alleles at an alpha level of 0.05 on the basis of the log-rank test.

\section{Regression models}

To elucidate the progression of SNHI in children with specific genotypes, linear regressions were performed on hearing levels in children of different ages with conclusive genotypes. All analyses were conducted using SAS software version 9.3 (SAS, Cary, NC).

\section{RESULTS}

Results of newborn genetic screening for deafness

Of the 5,173 newborns, $82(1.6 \%)$ were identified by newborn genetic screening as having conclusive genotypes: 62 (1.2\%) with GJB2 p.V37I/p.V37I, 16 (0.3\%) with GJB2 p.V37I/ c. $235 \mathrm{delC}$, and $4(0.1 \%)$ with $\mathrm{m} .1555 \mathrm{~A}>\mathrm{G}$ (Table 1). A total of 839 newborns (16.2\%) were detected to segregate one GJB2 or SLC26A4 mutation, including $736(14.2 \%)$ heterozygous for GJB2 p.V37I, 50 (1.0\%) heterozygous for GJB2 c.235delC, 
and $53(1.0 \%)$ heterozygous for SLC26A4 c.919-2A>G. Seven newborns $(0.1 \%)$ were double heterozygous for GJB2 p.V37I and SLC26A4 c.919-2A>G. Given the high frequency of the GJB2 p.V37I allele among the Taiwanese population, ${ }^{21}$ it was assumed that the great majority of these heterozygous subjects are coincidental carriers, and no further audiological or genetic assessments were conducted except for those who failed newborn hearing screening. Similarly, we did not routinely perform audiological or genetic workups in subjects segregating a single GJB2 c.235delC allele, a single SLC26A4 c.919-2A>G allele, or a GJB2/SLC26A4 double heterozygous genotype, except in those who failed newborn hearing screening. In the four subjects with the $\mathrm{m} .1555 \mathrm{~A}>\mathrm{G}$ mutation, the mutation load was detected as "homoplasmy" by hybridization probe testing for all four subjects; this was later confirmed using a published restriction enzyme digestion method (data not shown). ${ }^{22}$

\section{Newborn hearing screening results in infants with conclusive genotypes}

Of the 5,173 newborns, 3,149 were screened using DPOAEs, with a referral (failure) rate of $3.9 \%(123 / 3,149)$, and 2,024 were

Table 1 Newborn genetic screening results among the 5,173 subjects

Genotype

GJB2

\begin{tabular}{lc} 
p.V37I/p.V37I & $62(1.2)$ \\
p.V37I/c.235delC & $16(0.3)$ \\
c.235delC/c.235delC & $0(0)$ \\
p.V37I/wt & $736(14.2)$ \\
c.235delC/wt & $50(1.0)$ \\
SLC26A4 & \\
c.919-2A>G/c.919-2A>G & $0(0)$ \\
c.919-2A>G/wt & $53(1.0)$ \\
Mitochondrial 12S rRNA & \\
$\quad$ (m.1555A>G) & $4(0.1)$ \\
Mutations in two genes & $7(0.1)$ \\
(GJB2 p.V37I/wt SLC26A4 c.919-2A>G/wt) & \\
No mutation identified & $4,245(82.1)$ \\
\hline wt, wild type. &
\end{tabular}

screened using AABR, with a referral rate of $2.1 \%(42 / 2,024)$. Among the 62 babies with GJB2 p.V37I/p.V37I, 37 and 25 underwent DPOAEs and AABR for newborn hearing screening, respectively; of these, 23 babies (62.2\%) and 9 babies (36.0\%) passed DPOAEs and AABR screening, respectively (Table 2). Although the pass rate of DPOAEs was higher than that of AABR, the difference between the two screening tools was not statistically significant $(P=0.07$, Fisher's exact test). Of the 16 babies with GJB2 p.V37I/c.235delC who underwent DPOAEs $(n=11)$ or AABR $(n=5)$ for newborn hearing screening, 8 babies $(72.7 \%)$ and 2 babies (40.0\%) passed DPOAEs and AABR screening, respectively. There was no statistically significant difference between the pass rates of the two screening tools ( $P=0.30$, Fisher's exact test). On the other hand, all four babies (100\%) with the m.1555A $>\mathrm{G}$ mutation passed newborn hearing screening with either DPOAEs or AABR. In total, 46 babies (56.1\%) with conclusive genotypes passed newborn hearing screening, indicating that newborn genetic screening for deafness could identify an additional subgroup of babies with potential hearing problems-a subgroup that was not detected using either DPOAEs or AABR.

\section{Kaplan-Meier estimations}

In total, the 62 subjects with GJB2 p.V37I/p.V37I, the 16 subjects with GJB2 p.V37I/c.235delC, and the 4 subjects with m.1555A>G underwent 158,35 , and 4 audiological assessments during the follow-up periods, respectively. Kaplan-Meier analyses were not performed on the audiological data of infants with the m.1555A $>$ G genotype because of the insufficient number of observations. As for the other two genotypes, Kaplan-Meier analyses showed the progression of SNHI with time in children with either genotype. Setting $25 \mathrm{dBHL}$ as the event, $72.4 \%$ of children with GJB2 p.V37I/p. V37I and $80.0 \%$ of children with GJB2 p.V37I/c.235delC were estimated to have hearing levels $<25 \mathrm{dBHL}$ at 3 months (Figure 1). Among children with GJB2 p.V37I/p.V37I and those with GJB2 p.V37I/c.235delC, the percentages with hearing levels $<25 \mathrm{dBHL}$ decreased to 48.7 and $48.6 \%$, respectively, at 3 years, and decreased further to 41.0 and $45.7 \%$, respectively, at 5 years. There was no significant difference

Table 2 Newborn hearing screening results in the 82 subjects with conclusive genotypes on genetic screening

\begin{tabular}{|c|c|c|c|c|}
\hline \multirow[b]{2}{*}{ Genotype } & \multirow[b]{2}{*}{ NHS tool } & \multirow{2}{*}{$\begin{array}{c}\text { Subjects passing } \\
\text { NHS }(n)\end{array}$} & \multirow{2}{*}{$\begin{array}{c}\text { Subjects failing } \\
\text { NHS }(n)\end{array}$} & \multirow[b]{2}{*}{ Pass rate $(\%)$} \\
\hline & & & & \\
\hline \multirow[t]{2}{*}{ p.V37I/p.V37I } & DPOAEs $(n=37)$ & 23 & 14 & 62.2 \\
\hline & Total $(n=62)$ & 32 & 30 & 51.6 \\
\hline \multirow[t]{2}{*}{ p.V37l/c.235delC } & DPOAEs $(n=11)$ & 8 & 3 & 72.7 \\
\hline & $\operatorname{AABR}(n=5)$ & 2 & 3 & 40.0 \\
\hline \multicolumn{5}{|c|}{ Mitochondrial 125 rRNA } \\
\hline \multirow[t]{3}{*}{$(\mathrm{m} .1555 \mathrm{~A}>\mathrm{G})$} & DPOAEs $(n=2)$ & 2 & 0 & 100.0 \\
\hline & $\operatorname{AABR}(n=2)$ & 2 & 0 & 100.0 \\
\hline & Total $(n=4)$ & 4 & 0 & 100.0 \\
\hline Total $(n=82)$ & & 46 & 36 & 56.1 \\
\hline
\end{tabular}

AABR, automated auditory brainstem response; DPOAEs, distortion-product otoacoustic emissions; NHS, newborn hearing screening. 
in the progression of SNHI between the two groups (Cox hazard ratio, $0.89(95 \% \mathrm{CI}, 0.54-1.46) ; P=0.64)$. This is consistent with the results of a previous study showing that the severity of SNHI did not differ between patients with these two genotypes. ${ }^{23}$

When $40 \mathrm{dBHL}$ was set as the event, $80.4 \%$ of children with GJB2 p.V37I/p.V37I and $85.7 \%$ of children with GJB2 p.V37I/ c. 235delC were estimated to have hearing levels $<40 \mathrm{dBHL}$ at 3 months (Figure 2). Among children with GJB2 p.V37I/p.V37I and those with GJB2 p.V37I/c.235delC, the percentages with hearing levels $<40 \mathrm{dBHL}$ decreased to 76.6 and $80.0 \%$, respectively, at 3 years, and decreased further to 74.7 and $80.0 \%$, respectively, at 5 years. Again, there was no significant difference in the progression of SNHI between the two groups (Cox hazard ratio, 0.79 (95\% CI, 0.35-1.76); $P=0.56)$. In summary, although many children with GJB2 p.V37I/p.V37I or p.V37I/ c.235delC developed SNHI that required adaptive listening strategies, only a few of them developed SNHI severe enough to necessitate the use of hearing aids.

\section{Linear regressions}

To determine the rate of progression of SNHI with the GJB2 p.V37I/p.V37I and p.V37I/c.235delC genotypes, we performed linear regression analysis of the relationship between the follow-up time (years) and the hearing level (dBHL) (Figure 3). The rate of SNHI progression in children with GJB2 p.V37I/p. V37I did not significantly differ from that in children with GJB2 p.V37I/c.235delC. When pooled together, the hearing level in children with GJB2 p.V37I/p.V37I or p.V37I/c.235delC was estimated to deteriorate at approximately $1 \mathrm{dBHL} /$ year (hearing level $(\mathrm{dBHL})=18.7+1.0 \times$ year $)$.

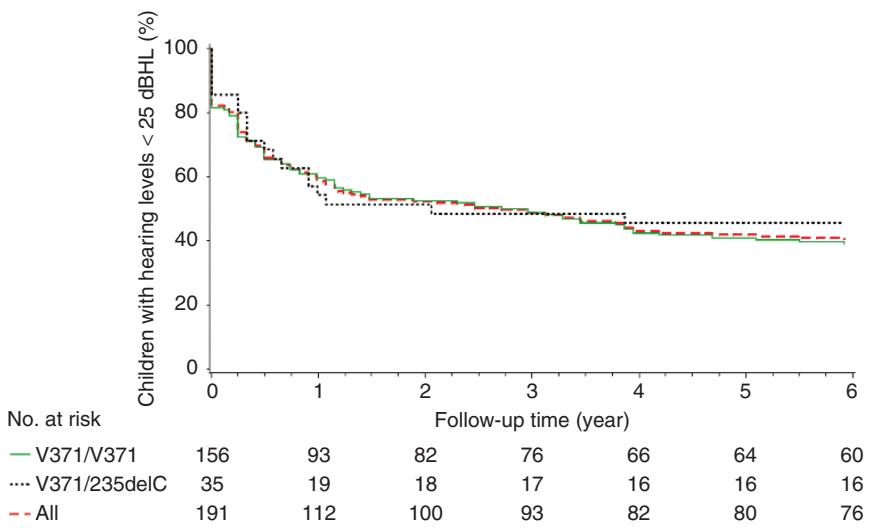

Figure 1 Kaplan-Meier analyses for a hearing level of 25 decibels (dBHL) in children with the GJB2 p.V37I/p.V37I and the GJB2 p.V37I/ c.235delC genotypes. Setting $25 \mathrm{dBHL}$ as the event, $72.4 \%$ of children with GJB2 p.V371/p.V37I and $80.0 \%$ of children with GJB2 p.V37l/c.235delC were estimated to have hearing levels $<25 \mathrm{dBHL}$ at 3 months. Among children with GJB2 p.V371/p.V37I and those with GJB2 p.V37I/c.235delC, the percentages with hearing levels $<25 \mathrm{dBHL}$ decreased to 48.7 and $48.6 \%$, respectively, at 3 years, and decreased further to 41.0 and $45.7 \%$, respectively, at 5 years. There was no significant difference in the progression of hearing loss between the two groups (Cox hazard ratio, 0.89 (95\% confidence interval, $0.54-1.46) ; P=0.64)$.

\section{DISCUSSION}

In this study we performed simultaneous hearing screening and genetic screening for deafness in 5,173 newborns, and we identified 82 babies $(1.6 \%)$ with conclusive genotypes on genetic screening, comprising $62(1.2 \%)$ with GJB2 p.V37I/p.V37I, $16(0.3 \%)$ with GJB2 p.V37I/c.235delC, and 4 (0.1\%) with m.1555A>G. Consistent with our previous study, ${ }^{15}$ a certain percentage $(56.1 \%)$ of these 82 babies passed newborn hearing screening, indicating that newborn genetic screening for deafness could identify an additional subgroup of babies with potential hearing problems that could not be detected by UNHS. We then conducted a longterm observational study (up to 6 years) to record the longitudinal auditory outcomes in these babies with conclusive genotypes; that study demonstrated progressive SNHI in children with the GJB2 p.V37I/p.V37I and p.V37I/c.235delC genotypes, which deteriorated approximately $1 \mathrm{dBHL} /$ year. Adding to our previous study that confirmed the utility of newborn genetic screening for deafness in identifying infants with slight/mild SNHI not targeted by conventional UNHS, ${ }^{15}$ the current study provided further evidence that genetic screening could also help identify children with late-onset or progressive SNHI frequently missed by UNHS.

Despite the utility of newborn genetic screening for deafness in identifying infants with slight/mild SNHI and those with late-onset/progressive SNHI, the integration of genetic screening into UNHS might raise new challenges and controversies. Ethical issues that might emerge with the implementation of newborn genetic screening for deafness include risks of discrimination or stigmatization, respect for a person's autonomy to make his or her own decisions, and undue parental anxiety for the health of their children. ${ }^{24}$ Socioeconomic consequences

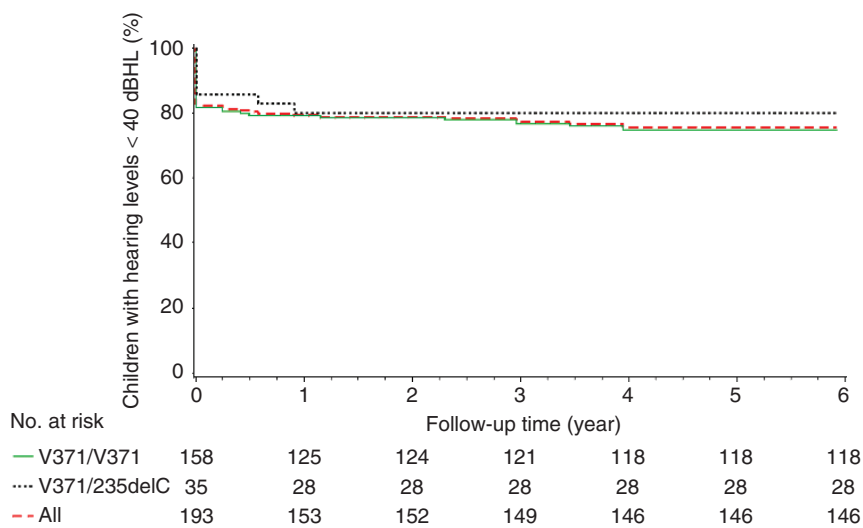

Figure 2 Kaplan-Meier analyses for a hearing level of $\mathbf{4 0}$ decibels (dBHL) in children with the GJB2 p.V37I/p.V37I and the GJB2 p.V371/ c.235delC genotypes. Setting $40 \mathrm{dBHL}$ as the event, $80.4 \%$ of children with GJB2 p.V37I/p.V37I and $85.7 \%$ of children with GJB2 p.V371/c.235delC were estimated to have hearing levels $\angle 40 \mathrm{dBHL}$ at 3 months. Among children with GJB2 p.V371/p.V371 and those with GJB2 p.V371/c.235delC, the percentages with hearing levels $<40 \mathrm{dBHL}$ decreased to 76.6 and $80.0 \%$, respectively, at 3 years, and decreased further to 74.7 and $80.0 \%$, respectively, at 5 years. There was no significant difference in the progression of sensorineural hearing impairment between the two groups (Cox hazard ratio, 0.79 (95\% confidence interval, 0.35-1.76); $P=0.56$ ). 
that might ensue include the necessity to modify or expand the infrastructure and human resources of the health-care system to support testing, counseling, education, treatment, and follow-up. Among various deafness mutations, screening for the maternally inherited m.1555A $>\mathrm{G}$ mutation among the general population seems to be the least controversial. As shown in this and other studies, m.1555A $>\mathrm{G}$ seems to be prevalent across different populations, ranging from 0.1 to $0.2 \% .^{18,25}$ Because permanent profound SNHI might develop in individuals carrying $\mathrm{m} .1555 \mathrm{~A}>\mathrm{G}$, with a penetrance close to $100 \%$ following standard therapeutic doses of aminoglycoside antibiotics, it has been suggested that $\mathrm{m} .1555 \mathrm{~A}>\mathrm{G}$ should be screened in all pregnant women to reduce aminoglycoside-associated hearing loss in newborns. ${ }^{26}$ As for other deafness mutations, however, more knowledge regarding their clinical features might be necessary to justify genetic screening in the general population.

In contrast to previous studies that reported only cross-sectional or short-term observations of newborn genetic screening for deafness, ${ }^{16-18}$ the strength of this study lies in that we prospectively performed comprehensive, long-term audiometric evaluations up to 6 years in babies with conclusive genotypes. These long-term observations are valuable in delineating the clinical features of the corresponding mutations. Specifically, to our knowledge, we documented for the first time in the literature the longitudinal auditory features of GJB2 p.V37I in a cohort recruited from the general population instead of from a diseased population. Owing to its high allele frequency (up to about 10\%) among East Asian populations, GJB2 p.V37I was regarded as a benign polymorphism in some earlier reports. ${ }^{21,27}$ However, biochemical and electrophysiological studies of cell lines showed that $\mathrm{p}$.V37I reduced the oligomerization efficiency of Cx26, the protein encoded by GJB2, and significantly compromised the channel function of gap junctions. ${ }^{28}$ Consistent with the experiments in cell lines, several clinical studies supported that homozygosity for p.V37I is associated with mild to moderate SNHI in humans. ${ }^{23,29-32}$ Analyses based on serial

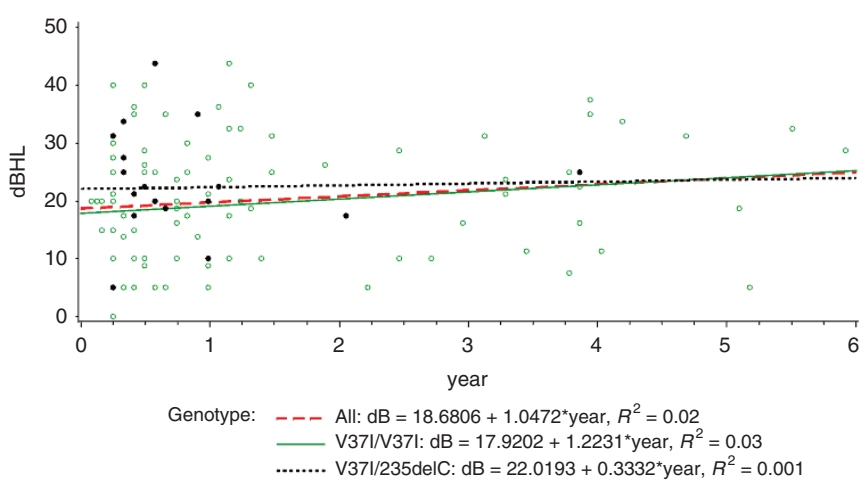

Figure 3 Linear regressions of hearing levels in children with the GJB2 p.V37I/p.V37I and the GJB2 p.V37I/c.235delC genotypes. Both children with GJB2 p.V37I/p.V37I and those with p.V37I/c.235delC showed progressive sensorineural hearing impairment with time, and the rate of progression did not differ significantly between the two groups. When pooled together, the hearing level in children with GJB2 p.V371/p.V37I or p.V37I/c.235delC was estimated to deteriorate at approximately 1 decibel hearing level $(\mathrm{dBHL}) /$ year (hearing level $(\mathrm{dBHL})=18.7+1.0 \times$ year). audiometric data in children with bi-allelic GJB2 mutations further revealed that, among a wide variety of different GJB2 mutations, progression of SNHI was particularly common among carriers of p.V37I either in homozygosity or in compound heterozygosity with another GJB2 mutation. ${ }^{9}$ Interestingly, a recent study in China reported five subjects homozygous for p.V37I who demonstrated normal hearing at the age of 20-30 years, ${ }^{33}$ suggesting incomplete penetrance of the auditory phenotypes. Because at least 5 million East Asians are estimated to be homozygous for p.V37I, it is crucial to elucidate the phenotypic spectrum and progression associated with this mutation from the perspectives of public health and clinical medicine. Our results in this study showed that subjects with p.V37I in homozygosity or in compound heterozygosity with c.235delC developed progressive SNHI at a rate of approximately $1 \mathrm{dBHL} /$ year. At the age of 5 years, $~ 50 \%$ of these subjects might need adaptive listening strategies for daily life (e.g., at school), and 20-25\% might even require hearing aids.

However, the results of this study should be interpreted and extrapolated with caution. First, only commonly known deafness-associated mutations were included in the screening panel. This might lead to a false assurance for individuals who actually segregate rare deafness mutations. This limitation, however, might be overcome with the decreasing cost of high-throughput DNA sequencing, which has recently been proven as an effective tool for addressing hereditary hearing impairment by simultaneously screening a large number of deafness genes. ${ }^{34,35}$ Indeed, a recent study showed that more than $70 \%$ of parents are interested in whole-genome sequencing for their newborns, ${ }^{36}$ and it was predicted that in the next decade large-scale sequencing for all healthy babies at birth could be plausible and might change the practice of conventional newborn screening programs ${ }^{37}$ Second, as participants in this study were recruited from 2009 to 2015, only a limited number of subjects completed their follow-up for more than 5 years. Therefore, it might be difficult to use our regression model to predict the hearing outcomes after the age of 5 years in subjects with conclusive genotypes. Further documentation of auditory phenotypes in subjects with specific genotypes is warranted to refine this model to predict long-term hearing outcomes. Third, although our results demonstrated progressive SNHI in children with the GJB2 p.V37I/p.V37I and p.V37I/c.235delC genotypes, hearing levels varied significantly among individuals at different ages, as shown in Figure 3. It is conceivable that other genetic or environmental factors might modulate the auditory phenotypes associated with p.V37I. For a more precise prediction of the auditory outcomes in these individuals, further studies are needed to elucidate the interplay between p.V37I and these factors.

Notably, the additional benefit of integrating genetic screening into the UNHS program might depend on the tools used for hearing screening. Although there was no statistically significant difference between the two screening tools, the pass rate of DPOAEs in newborns with GJB2 p.V37I/p.V37I or p.V37I/c.235delC was higher than that of AABR (Table 2), 
indicating that DPOAEs might be less sensitive than AABR in detecting potential hearing problems in newborns with these two genotypes. For populations with a high prevalence of these two genotypes, AABR might be the recommended hearing screening tool if genetic screening is not available.

In addition to hearing screening and genetic screening, screening of newborns for cytomegalovirus (CMV) infection has been proposed for early identification of infected infants who are at risk of developing SNHI. ${ }^{38}$ It is estimated that the prevalence of congenital CMV infection in developed countries is $0.58 \%$ and might contribute to $2-18 \%$ of cases with pediatric SNHI. ${ }^{39}$ The feasibility of integrating congenital CMV screening into the UNHS program has been illustrated in a recent study conducted in the United Kingdom. ${ }^{40}$ Another pilot study is currently underway at our hospital to explore the feasibility and long-term cost-effectiveness of a comprehensive newborn screening program comprising simultaneous hearing, genetic, and CMV screenings at birth.

In conclusion, by performing simultaneous hearing and genetic screenings in a large newborn cohort and analyzing serial audiometric results up to 6 years in those with conclusive genotypes, our results delineated the longitudinal auditory outcomes of the highly prevalent GJB2 p.V37I mutation on a general population basis and confirmed the utility of newborn genetic screening in identifying infants with late-onset or progressive hearing impairment undetectable by newborn hearing screening. These findings help to inform future newborn screening policy and provide insights into risk assessment and genetic counseling for both the physicians and the parents of babies who screen positive for deafness mutations.

\section{ACKNOWLEDGMENTS}

This study was supported by research grants from the Ministry of Science and Technology of the Executive Yuan of Taiwan (MOST 102-2314-B-303-016-MY3) and National Taiwan University Hospital (NTUH 101-001848). The authors thank all subjects and their parents for participating in this study.

\section{DISCLOSURE}

The authors declare no conflict of interest.

\section{REFERENCES}

1. Morton CC, Nance WE. Newborn hearing screening-a silent revolution. NEngl J Med 2006;354:2151-2164.

2. Thompson DC, McPhillips H, Davis RL, Lieu TL, Homer CJ, Helfand M Universal newborn hearing screening: summary of evidence. JAMA 2001;286: 2000-2010.

3. Yoshinaga-Itano C, Sedey AL, Coulter DK, Mehl AL. Language of early- and later-identified children with hearing loss. Pediatrics 1998;102:1161-1171.

4. Barsky-Firkser L, Sun S. Universal newborn hearing screenings: a three-year experience. Pediatrics 1997;99:E4

5. Kennedy CR, McCann DC, Campbell MJ, et al. Language ability after early detection of permanent childhood hearing impairment. N Engl J Med 2006;354:2131-2141.

6. Johnson JL, White KR, Widen JE, et al. A multicenter evaluation of how many infants with permanent hearing loss pass a two-stage otoacoustic emissions/ automated auditory brainstem response newborn hearing screening protocol. Pediatrics 2005;116:663-672.
7. Young NM, Reilly BK, Burke L. Limitations of universal newborn hearing screening in early identification of pediatric cochlear implant candidates. Arch Otolaryngol Head Neck Surg 2011;137:230-234.

8. Hilgert N, Smith RJ, Van Camp G. Forty-six genes causing nonsyndromic hearing impairment: which ones should be analyzed in DNA diagnostics? Mutat Res 2009;681:189-196.

9. Chan DK, Schrijver I, Chang KW. Connexin-26-associated deafness: phenotypic variability and progression of hearing loss. Genet Med 2010;12:174-181.

10. Chan DK, Chang KW. GJB2-associated hearing loss: systematic review of worldwide prevalence, genotype, and auditory phenotype. Laryngoscope 2014;124:E34-E53.

11. Miyagawa M, Nishio SY, Usami S; Deafness Gene Study Consortium. Mutation spectrum and genotype-phenotype correlation of hearing loss patients caused by SLC26A4 mutations in the Japanese: a large cohort study. J Hum Genet 2014;59:262-268.

12. Kokotas H, Petersen MB, Willems PJ. Mitochondrial deafness. Clin Genet 2007;71:379-391.

13. Minami SB, Mutai $H$, Nakano A, et al. GJB2-associated hearing loss undetected by hearing screening of newborns. Gene 2013;532:41-45.

14. Kim BG, Shin JW, Park HJ, Kim JM, Kim UK, Choi JY. Limitations of hearing screening in newborns with PDS mutations. Int J Pediatr Otorhinolaryngol 2013;77:833-837.

15. Wu CC, Hung CC, Lin SY, et al. Newborn genetic screening for hearing impairment: a preliminary study at a tertiary center. PLoS One 2011; 6:e22314

16. Zhang Z, Ding $W$, Liu $X$, et al. Auditory screening concurrent deafness predisposing genes screening in 10,043 neonates in Gansu province, China. Int J Pediatr Otorhinolaryngo/ 2012;76:984-988.

17. Yao GD, Li SX, Chen DL, et al. Combination of hearing screening and genetic screening for deafness-susceptibility genes in newborns. Exp Ther Med 2014;7:218-222.

18. Zhang J, Wang $P$, Han B, et al. Newborn hearing concurrent genetic screening for hearing impairment-a clinical practice in 58,397 neonates in Tianjin, China. Int J Pediatr Otorhinolaryngo/ 2013;77:1929-1935.

19. Wu CC, Chen PJ, Chiu YH, Lu YC, Wu MC, Hsu CJ. Prospective mutation screening of three common deafness genes in a large Taiwanese Cohort with idiopathic bilateral sensorineural hearing impairment reveals a difference in the results between families from hospitals and those from rehabilitation facilities. Audiol Neurootol 2008;13:172-181.

20. Wu CC, Lee YC, Chen PJ, Hsu CJ. Predominance of genetic diagnosis and imaging results as predictors in determining the speech perception performance outcome after cochlear implantation in children. Arch Pediatr Adolesc Med 2008;162:269-276.

21. Hwa HL, Ko TM, Hsu CJ, et al. Mutation spectrum of the connexin 26 (GJB2) gene in Taiwanese patients with prelingual deafness. Genet Med 2003;5: 161-165.

22. Wu CC, Chiu YH, Chen PJ, Hsu CJ. Prevalence and clinical features of the mitochondrial m.1555A>G mutation in Taiwanese patients with idiopathic sensorineural hearing loss and association of haplogroup F with low penetrance in three families. Ear Hear 2007;28:332-342.

23. Huang S, Huang B, Wang G, Yuan Y, Dai P. The relationship between the p.V37I mutation in GJB2 and hearing phenotypes in Chinese individuals. PLoS One 2015;10:e0129662.

24. Dhondt JL. Expanded newborn screening: social and ethical issues. J Inherit Metab Dis 2010;33(Suppl 2):S211-S217.

25. Bitner-Glindzicz $M$, Pembrey $M$, Duncan $A$, et al. Prevalence of mitochondrial 1555A-> G mutation in European children. N Engl J Med 2009;360: 640-642.

26. Linden Phillips $L$, Bitner-Glindzicz $M$, Lench $N$, et al. The future role of genetic screening to detect newborns at risk of childhood-onset hearing loss. Int J Audiol 2013;52:124-133.

27. Wattanasirichaigoon $D$, Limwongse $C$, Jariengprasert C, et al. High prevalence of V37I genetic variant in the connexin-26 (GJB2) gene among non-syndromic hearing-impaired and control Thai individuals. Clin Genet 2004;66:452-460.

28. Jara O, Acuña R, García IE, et al. Critical role of the first transmembrane domain of $\mathrm{C} \times 26$ in regulating oligomerization and function. Mol Biol Cell 2012;23:3299-3311.

29. Snoeckx RL, Huygen PL, Feldmann D, et al. GJB2 mutations and degree of hearing loss: a multicenter study. Am J Hum Genet 2005;77:945-957.

30. Pollak A, Skórka A, Mueller-Malesińska M, et al. M34T and V37l mutations in GJB2 associated hearing impairment: evidence for pathogenicity and reduced penetrance. Am J Med Genet A 2007;143A:2534-2543. 


\section{ORIGINAL RESEARCH ARTICLE}

31. Tsukada K, Nishio S, Usami S; Deafness Gene Study Consortium. A large cohort study of GJB2 mutations in Japanese hearing loss patients. Clin Genet 2010;78:464-470.

32. Kim SY, Park G, Han KH, et al. Prevalence of $p$. V37I variant of GJB2 in mild or moderate hearing loss in a pediatric population and the interpretation of its pathogenicity. PLoS One 2013;8:e61592.

33. Chai $Y$, Chen $D$, Sun $L$, et al. The homozygous p.V37I variant of GJB2 is associated with diverse hearing phenotypes. Clin Genet 2015;87:350-355.

34. Shearer AE, DeLuca AP, Hildebrand MS, et al. Comprehensive genetic testing for hereditary hearing loss using massively parallel sequencing. Proc Natl Acad Sci USA 2010;107:21104-21109.

35. Brownstein Z, Friedman LM, Shahin H, et al. Targeted genomic capture and massively parallel sequencing to identify genes for hereditary hearing loss in Middle Eastern families. Genome Biol 2011;12:R89.
36. Goldenberg AJ, Dodson DS, Davis MM, Tarini BA. Parents' interest in whole-genome sequencing of newborns. Genet Med 2014;16:78-84.

37. Knoppers BM, Sénécal K, Borry P, Avard D. Whole-genome sequencing in newborn screening programs. Sci Trans/ Med 2014;6:229cm2.

38. Boppana SB, Ross SA, Shimamura M, et al.; National Institute on Deafness and Other Communication Disorders CHIMES Study. Saliva polymerasechain-reaction assay for cytomegalovirus screening in newborns. N Engl J Med 2011:364:2111-2118.

39. Goderis J, De Leenheer E, Smets K, Van Hoecke H, Keymeulen A, Dhooge I. Hearing loss and congenital CMV infection: a systematic review. Pediatrics 2014;134:972-982.

40. Kadambari S, Luck S, Davis A, et al. Evaluating the feasibility of integrating salivary testing for congenital CMV into the Newborn Hearing Screening Programme in the UK. Eur J Pediatr 2015;174:1117-1121. 